\title{
Research on Singing Mechanism Application to College Music Teaching based on Intelligent Electronic Assistant
}

\author{
Cheng Gui Song \\ Teacher's College, Beihua University, Jilin City, China
}

\begin{abstract}
Music is one of disciplines with special arts, because under the constraints of objective factors people can derive from the physical feeling, either the acquisition of knowledge or grasp of skills. This paper reveals the issues met more easily in music teaching and nature behind the phenomenon through the research on the mechanism characteristics of three most critical and basic elements.
\end{abstract}

\section{General instruction}

Any construction and development of a discipline or an art categories, not just experience or just stay in the morphological level, only to find and exploit their experience or morphological diversity of the nature of the housing, and put them up to the scientific nature of contact systematic theoretical level, in order to bring about a qualitative change, and to promote their better development. And promote the construction and development of any one discipline or art categories ultimate factors, usually not only from the discipline or disciplines of the arts itself. But by the latest scientific research into the knowledge or discipline or other kinds of art in order to constantly improve, and even innovation. Throughout the history of vocal music from the heritage point of view, in fact, is the history of vocal music teaching development. "Oral teaching" is the main mode of traditional vocal music teaching; experience, feel, experience is the main theoretical basis of traditional vocal music teaching mode granted knowledge. So are bound to make an analogy, inspired imitation become the main means of teaching. Admittedly, vocal arts as a music category has the particularity of a discipline, the teaching mode and teaching methods irreplaceable leading role in the long history of traditional vocal music teaching development, so far it is still our the main mode and means of vocal teaching on. But the same can not be avoided, rely mainly on experience as a theoretical basis for the grant of knowledge and inspiration as a way to mimic the main learning and teaching methods are bound to exist its limitations and shortcomings.

This deficiency also embodied in both the grasp of vocal technique and tradition, and the vocal interpretation of the phenomenon is from their own personal experience. Thus a so-called blind men feeling the elephant, touch what like result. Vocal learning and teaching can bring some obstacles. In recent centuries, with the development of scientific and technological level of exploration and study of human anatomy in humans, acoustics, aerodynamics, etc. have made considerable progress. Many people many of whom are non-vocal music professionals in these areas will be thinking and research theory is applied to the exploration and study vocal learning occurs in various phenomena, for the traditional vocal music teaching philosophy injection of fresh blood, the traditional way of teaching vocal music and means full of new vitality.

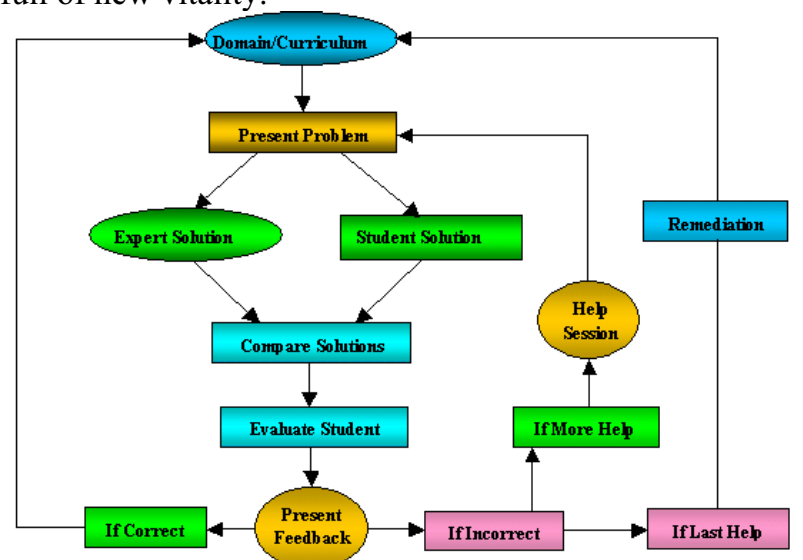

Figure 1. Intelligent Electronic Assistant for Music Teaching

\section{Incentive motivation "power source" mechanism singing in touch with the difference between breathing and respiratory}

As the physiological needs, the purpose of breathing human organism activity is to provide the necessary oxygen. As for the singing, the breathing is to provide a sound source of power. So, Breath and breathing our natural state of physical contact in essence they both have a difference on the purpose and methods. The perspective 
of contact is the main organs involved in singing breathing consistent with the natural breathing time. Lung is still stored gas exchange and organs involved in the expansion and contraction of the chest organs are still the main intercostals' muscles, diaphragm, abdominal, etc., from this point of view, which is essentially the physiological breathing is consistent.

But for singing breath, its main purpose is to give the vibration of the vibrator (vocal) provide energy. This makes breathing singing are essentially different in purpose and physiological breathing. Secondly, singing respiratory system must meet the requirements can control air pressure changes, and the whole story of this pressure output, the size, the length of duration must meet specific requirements when singing, and this requirement is by controlling the aspiratory muscles and breathing together with the active participation of the respiratory muscles while the movement to achieve, which makes breathing and singing breathe a certain physiological differences in the way. Finally, due to the complex structure of human organs and tissues and among inextricably linked, so consider how to better control of breathing while singing does not affect the normal operation of the vocal cords. So, what kind of breathing control can be achieved either arbitrarily controlled air pressure without affecting the normal operation of the vocal cords.

To better understand the singing breath control process and the principle of operation of this mechanism, we can also be inspired by observing the daily life of certain phenomena. For example, many people have seen or played in a child made of elastic rubber squeezes can be issued when the whistle toys. This toy is hollow, one end of the toy has a hole, the hole can cause vibration inlaid with reeds. When you squeezes it, the toy will be through the air vent whistle, whistle hole in the air due to the role of the reed vibrate and make noise. You squeeze force, the more rapidly, and its sound is bigger and shorter, whereas the smaller sound, the longer duration. This is our reason for respiratory control when singing is roughly the same. Specific play like elastic body from our aspiratory muscles holding function, and if pressing it by hand muscles our breath when we extradited, the air is squeezed toys after by whistle vent and cause the reed (as vocal cords) vibration. Under other conditions the same conditions, the greater the pressure, the reed vibrations are more severe, the sound will be. By doing a little toy testing, may make it easier for you to understand that we control the breathing process characteristics at the time of the singing mechanism.

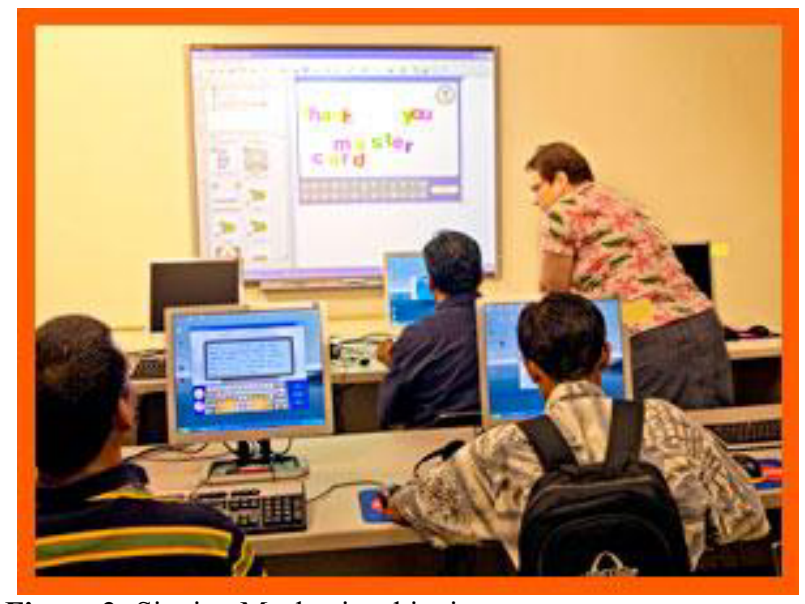

Figure 2. Singing Mechanism bionic agency

\section{The point of view mainly vocal mechanism muscle}

Larynx is located in the anterior middle, under the upper end of the trachea, hyoid; on through the hypo pharynx, below the lungs through the trachea connectivity. And we usually refer to it through a series of vocal ligaments, cartilage and other tissue attachment in the throat, and the throat is divided into several areas. First, the next vocal structure itself. Also the sound of vocal folds is lips and other sound.

There are two folds sound, so each one and the same at a level surface. Each sound folds itself divided down into two parts, the upper part is called the plicate, also called false vocal cords, the following section is called the lower folds, folds under what we call the usual sense of the vocal cords. False vocal cord in the normal pronunciation is not closed; it does not have a real sense of sound effects. The main role is to assist the true vocal cords false vocal cord closure.

Control the position of the larynx down and relatively moderate stability is necessary for vocal music. This requirement is in singing has its practical significance. Larynx bit down and remained stable from the point of view mainly vocal mechanism muscle affects the thyroid cartilage down and remain stable. The thyroid cartilage causing the vocal cords to expand longitudinally elongated tension. When the vocal tension expanding, we control the breath through to its greater pressure, the frequency of vibration of the vocal cords will increase, so that the vocal cords can be issued with the necessary conditions for a higher tone. The singing, the ability to sing has a good high and low quality sound, good or bad throat decisive position control. On the other hand, from the human voice classification analysis, the level and type of voice when singing larynx position has a great contact. Different voice types must have swallowed a certain length of pipe as a variable corresponding to the resonance pipe. The variable resonance tube shape has a direct and throat "proper position" display relevant. Essential meaning of the above is actually a good control of throat singing bits of lies. 
If there is some sort of principle can prove, using only breath can make glottal vibration, or even be able to breath through the glottis partially closed, then I believe that makes learning to sing students believe should not be excessive use of throat singing muscle strength will be easier. Bell Noli effect is such a principle. Implications of this principle to bring vocal music and teaching will be very positive. Bell Noli aerodynamic effect is an important principle, its use in today's everyday life everywhere. The lifting force aircraft, sprayer required suction force, are a manifestation of this principle. At the same time it is also an element of wind instruments vibrating body vibration. To better understand the singing breath control process and the principle of operation of this mechanism, we can also be inspired by observing the daily life of certain phenomena. For example, many people have seen or played in a child made of elastic rubber squeezes can be issued when the whistle toys. This toy is hollow, one end of the toy has a hole, the hole can cause vibration inlaid with reeds. When you squeezes it, the toy will be through the air vent whistle, whistle hole in the air due to the role of the reed vibrate and make noise. You squeeze force, the more rapidly, and its sound is bigger and shorter, whereas the smaller sound, the longer duration. This is our reason for respiratory control when singing is roughly the same. Specific play like elastic body from our aspiratory muscles holding function, and if pressing it by hand muscles our breath when we extradited, the air is squeezed toys after by whistle vent and cause the reed (as vocal cords) vibration. Under other conditions the same conditions, the greater the pressure, the reed vibrations are more severe, the sound will be. By doing a little toy testing, may make it easier for you to understand that we control the breathing process characteristics at the time of the singing mechanism.

In fact, we pass a simple test can demonstrate that this principle characteristics. We'll twin vertical piece of paper each hand holding, when we put two sheets of paper on the lips, blowing when the two pieces of paper in the middle, perhaps you will think these two sheets of paper will be blown away from each other. In fact, just the opposite result, but by not separate two pieces of paper together, and began to vibrate. This phenomenon is caused by the Bell Noli effect. Its principle is as follows: before blowing, the air pressure and the air pressure in the middle of two sheets of paper the two sides of the same, in the absence of external forces, the paper original state. And when the middle of the two pieces of paper blowing in the middle of two paper because air pressure is reduced reasons, so the pressure on both sides of two pieces of paper began to push the two pieces of paper together. Two pieces of paper each squeeze of the extent and time will also change by the middle, when you stop blowing, two pieces of paper and re-open. Until next time blowing, this phenomenon will begin the process of circulation.

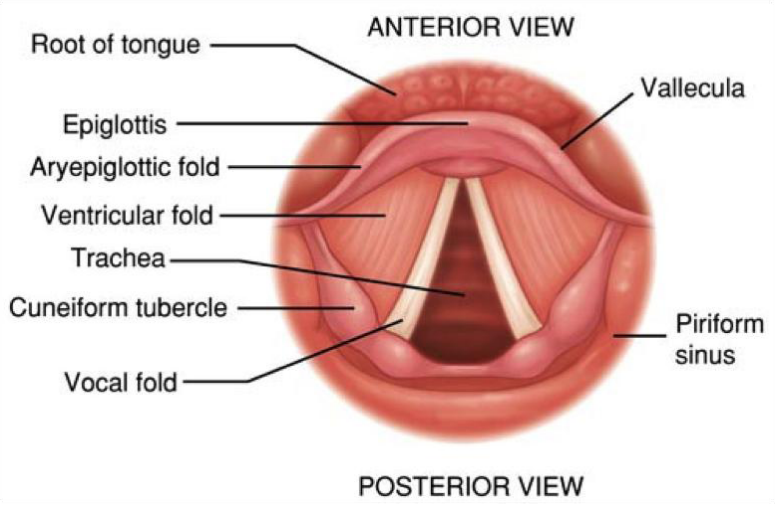

Figure 3. Vocal sound institutions and principles

\section{The same mechanism to force}

Prior to the structural characteristics of human resonance cavity and their effects on the sound emitted by the vocal cords to be concerned about, I think we should have the necessary knowledge of the relevant acoustic certain understanding. Because it will help us to a better understanding of "human acoustic instruments," the resonance body for sound effects.

The reason why the sound is generated and transmitted, because the vibration of a vibrating body under the action of some kind of energy, and this disturbed vibration between the vibrating body in the surrounding air molecules and molecules of the original balance of the pitch, so that the vibrating body is disrupting molecules lost like lake ripples like a stone into the sky to form a peripheral compression wave is formed. This wave is our auditory system has become a voice message is received after the treatment. Therefore, the sound waves transmitted in the form. For the size of the sound is concerned, the objective is to depend on the size of the vibrating body vibration amplitude, while the pitch is from the vibrating body vibration speed (frequency) of the decision.

For the "resonance" meaning, from the physical interpretation is: a physical cause another vibrating body vibration on the same or similar frequency vibration phenomenon. And when the same or very close to the resonance frequency of the vibration between the vibrating body and the bodies are forced, the forced vibration causes the vibrating body and the combined energy of the body itself to produce more than the vibration energy. This phenomenon is called the acoustic "resonance." When the two vibrating the vibrating body is not the same frequency, mutual interference between the two may even conflict with the energy and quickly disappears.

For singing is concerned, we want to do is to be issued after the sound of the vocal cords in the body resonates with our body resonance effect through amplification and modification of maximum efficiency, making it a beautiful, touching song. For the vocal sound experiments, humans have very clear sound to confirm the basis of the vocal cords in the body from the volume 
is very weak; from the tone, the resonance effect after modification and songs have a single multi-comparison. So, if in the singing voice in order to have a more perfect, we must effectively resonate with the aid of this tool will be expanded vocal sound in volume, embellished on the sound. This is a subjective desire of the singer. But also in the production of any musical instrument makers, the need to pursue the reason resonance effect resonance body. This requirement is in singing has its practical significance. Larynx bit down and remained stable from the point of view mainly vocal mechanism muscle affects the thyroid cartilage down and remain stable. The thyroid cartilage causing the vocal cords to expand longitudinally elongated tension. When the vocal tension expanding, we control the breath through to its greater pressure, the frequency of vibration of the vocal cords will increase, so that the vocal cords can be issued with the necessary conditions for a higher tone. The singing, the ability to sing has a good high and low quality sound, good or bad throat decisive position control. On the other hand, from the human voice classification analysis, the level and type of voice when singing larynx position has a great contact. Different voice types must have swallowed a certain length of pipe as a variable corresponding to the resonance pipe.

The resonance is not the source of all the wonderful sounds of it? If you put this guiding ideology into singing practice, you will find just blindly through the pursuit of sound resonance effect to achieve the perfect idea is not practical. Even if your mouths do well, you are also in accordance with the respective cavity resonance teacher asked to do very well. The actual effect is not the person singing wishful. Do not forget, singing is a process of mutual cooperation and efforts between the various structural elements of the vocal instrument. First, in order to let the resonance effect in singing to achieve better results, you have to make your vibrating body - the vocal cords (first vibrating body) itself play better results. For singing, the resonance effect is just an effect of feeling rather than a groundless effect. In other words, the first vibrating body - the vocal cords themselves, though not the last beautiful singing only the founder, but its role is first and foremost, can not be ignored. There are two folds sound, so each one and the same at a level surface. Each sound folds itself divided down into two parts, the upper part is called the plicate, also called false vocal cords, the following section is called the lower folds, folds under what we call the usual sense of the vocal cords.

Perhaps no other discipline would like vocal, as the presence of so many of the divergent, even opposing views on the grasp of skills and knowledge. "Miss is as good of a thousand miles," the phrase used in vocal learning might be fitting. Vocal music is an art, but it is first gate technology. Good and bad judgment on the level of technology that we may be able to simply use the "yes" and "none" to a conclusion. But for technology, learning and heritage, it must reach the "know, but also know why" requirements.
In my ten years of vocal learning process, have experienced a lot of hardships, but also through a lot of detours, the reason, in addition to the constraints of objective factors, but mainly because of their lack of contact on the back of the most essential understanding of vocal phenomenon; and precisely this understanding is based on cognitive mechanisms singing on.

\section{References}

1. Liu Jiusi. On the Italian bel canto vocal performance. Qiqihar Teachers College School Newspaper, 1989.

2. Qiao Ailing Discussion and Practice of Vocal Music Teaching. Xinghai Conservatory of Music 26(2002)

3. YAN Jing-yu regulate its use singing resonance. Qilu Art Gallery, 58(2002)

4. Zhang Xian sound technical and artistic resolve singing. People's Music, 154(2005)

5. Cuiquan Xin Singing resonance cavity resonance and three practical exercises. Xinghai Conservatory of Music School Newspaper, 25(2001)

6. Xia Xianping vocal cords, glottis and vocal. Biology Bulletin, 198(2012)

7. Zhu Jiming, Rick Chan, Lubbe real larynx anatomical study. Shanxi: Anatomy, 213(2009) 